\title{
Nonlinear Decentralized Disturbance Attenuation Excitation Control via New Recursive Design for Multi-Machine Power Systems
}

\author{
Qiang Lu, Senior Member, IEEE, Shengwei Mei, Member, IEEE, Wei Hu, Student Member, IEEE, \\ Felix F. Wu, Fellow, IEEE, Yixin Ni, Senior Member, IEEE, and Tielong Shen
}

\begin{abstract}
In this paper, a new nonlinear decentralized disturbance attenuation excitation control for multi-machine power systems is proposed based on recursive design without linearization treatment. The proposed controller improves system robustness to dynamic uncertainties and also attenuates bounded exogenous disturbances on the system in the sense of $L_{2}$-gain [1]. Computer test results on a 6-machine system show clearly that the proposed excitation control strategy can enhance transient stability of power systems more effectively than other excitation controllers.
\end{abstract}

Index Terms-Decentralized control, disturbance attenuation, nonlinear system, power system stability, recursive design.

\section{INTRODUCTION}

$\mathbf{P}$ OWER systems are large scale, distributed and highly nonlinear systems with complicated and fast transients. The random and exogenous disturbances especially short-circuit faults make the system stability problem even more severe. The existing linear control strategies, such as PID control, PSS (Power System Stabilizer) and LOEC (Linear Optimal Excitation Control) [2]-[4] have some drawbacks because their designs are based on linearized system models at one or more operating points and verified by nonlinear system simulation. Under certain unforeseen large disturbances the performance of linear controllers might not be satisfactory because of highly nonlinear nature of real power systems. Besides the selection of base operation points and large disturbances is quite empirical. With the application of differential geometric approach to power systems by Lu et al. [5], [6], many fruitful results have been achieved in nonlinear control of power systems [7]-[9]. It is noticed that nonlinear control designed based on differential geometric approach has much better effect than their linear counterparts mentioned above on enhancing power system

Manuscript received July 12, 2000; revised June 26, 2001. This work was supported by the Natural Science Foundation of China (Grant NSFC-59837270), the National Key Basic Research Special Foundation (Grant G1998020309) and the NEDO International Joint Research Grant.

Q. Lu, S. Mei, and W. Hu are with the Department of Electrical Engineering, Tsinghua University, Beijing, 100084, China.

F. F. Wu and Y. Ni are with the Department of Electrical and Electronic Engineering, The University of Hong Kong, Hong Kong SAR.

T. Shen is with the Department of Mechanical Engineering, Sophia University, Tokyo, Japan.

Publisher Item Identifier S 0885-8950(01)09430-5. large disturbance stability [10]. However, these nonlinear design approaches are generally based on mathematic models with fixed structure and parameters without considering uncertainties regarded as disturbances in modeling. In order to overcome the limitation and to enhance robustness of systems, modern nonlinear robust control has been applied to power systems and yielded attractive results [11]-[13]. This paper aims at developing a new nonlinear decentralized disturbance attenuation control for multi-machine power systems based on recursive design without linearization treatment. The proposed controller improves system robustness to dynamic uncertainties, measurement errors and also attenuates bounded exogenous disturbances on the system in the sense of $L_{2}$-gain [1].

The design of new controller involves two steps. First step is to establish robust model considering disturbances, and to select the regulation output such as tracking errors. The second step is to design the nonlinear robust controller to attenuate the influence of disturbances on the regulation output. Since power systems are interconnected and distributed over vast areas, the designed nonlinear robust control law should have decentralized feature, that is, the variables appeared in the expression of control law should be local measurements.

Indeed, a series of literature have discussed decentralized control of power systems [7], [8], [10]-[14], [17], however the research work on applying nonlinear decentralized and robust control to multi-machine systems is marginal. Significant contribution has been made in [15], [16], [19], in which nonlinear adaptive control methods are developed by using so-called back-stepping algorithm. Stimulated by [15], [16] and [19], this paper develops a new recursive design method, through which the dissipative inequality for excitation control of a multi-machine system is solved and the expression of nonlinear decentralized and the corresponding robust excitation control law for multi-power systems is derived.

The paper is arranged as follows. In Section II, nonlinear decentralized disturbance attenuation problem is defined with its standard format presented. In Section III, the system model is built up for multi-machine excitation robust control design. The author derives the nonlinear robust excitation control law using recursive design method explicitly. Computer test results of a multi-machine power system are given in Section IV with the conclusion drawn in Section V. 


\section{Nonlinear DeCEntralized Disturbance AtTenuation PROBLEM}

The definition of a nonlinear decentralized disturbance attenuation problem (NDDAP) is as follows.

Consider a large-scale nonlinear system composed of interconnected sub-systems $P_{i}(1 \leq i \leq n)$ and described as

$$
\left\{\begin{array}{l}
\dot{x}_{i 1}=f_{i 1}\left(x_{i}\right)+g_{i 1}(x)+S_{i 1}\left(x_{i}\right) w_{i 1} \\
\dot{x}_{i 2}=f_{i 2}\left(x_{i}\right)+g_{i 2}(x)+S_{i 2}\left(x_{i}\right) w_{i 2} \\
\vdots \quad \vdots \quad \vdots \\
\dot{x}_{i k-1}=f_{i(k-1)}\left(x_{i}\right)+g_{i(k-1)}(x)+S_{i(k-1)}\left(x_{i}\right) w_{i(k-1)} \\
\dot{x}_{i k}=f_{i k}\left(x_{i}\right)+g_{i k}(x) u_{i}+S_{i k}\left(x_{i}\right) w_{i k} \\
y_{i}=h\left(x_{i}\right) \quad(1 \leq i \leq n, 1 \leq j \leq k, 1 \leq k \leq m)
\end{array}\right.
$$

where

$$
\begin{aligned}
x_{i} & =\left(x_{i 1}, x_{i 2}, \ldots, x_{i k}\right)^{T} \in R^{k} ; \\
x & =\left(x_{1}^{T}, x_{2}^{T}, \ldots, x_{n}^{T}\right)^{T} \in R^{n \times k} ;
\end{aligned}
$$

$x$ is the state vector, $u_{i} \in R$ is the control input;

$$
w_{i}=\left(w_{i 1}, w_{i 2}, \ldots, w_{i k}\right)^{T} \in R^{k}
$$

is the exogenous disturbance; $y_{i} \in R^{p}$ is the regulation output; the functions $f_{i j}, g_{i j}$ and $S_{i j}$ are $C^{1}$ mapping with corresponding dimensions.

Problem: For system (1), the nonlinear decentralized disturbance attenuation problem is to construct $C^{1}$ state feedback controller $u_{i}=u_{i}\left(x_{i}\right), 1 \leq i \leq n$ such that for a prescribed positive number $\gamma$, the corresponding closed-loop system satisfy the following $L_{2}$-gain dissipative inequality [1]

$$
\begin{array}{r}
\int_{0}^{T}\|y\|^{2} d t \leq \gamma^{2} \int_{0}^{T}\|w\|^{2} d t+V\left(x_{0}\right) \\
\forall w \in L_{2}(0, T) \quad \forall T \geq 0
\end{array}
$$

where $V(\cdot)$ is a nonnegative storage function to be constructed, and $x_{0}$ is a initial state; $L_{2}(0, T)$ denotes $L_{2}$-space;

$$
w=\left(w_{1}^{T}, w_{2}^{T}, \ldots, w_{n}^{T}\right)^{T} \in R^{n \times k}
$$

is the exogenous disturbance; $\|\cdot\|$ denotes the Euclidean norm of a vector; and the $L_{2}$-space is defined as

$$
\begin{aligned}
& L_{2}(0, T) \\
& \quad=\left\{w \mid w:[0, T) \rightarrow R^{n \times k}, \text { and } \quad \int_{0}^{T}\|w\|^{2} d t<+\infty\right\} .
\end{aligned}
$$

Inequality (2) means that the $L_{2}$-gain of the system from disturbance $w$ to output $y$ is less than or equal to the prescribed number $\gamma$, which also means that the influence of disturbance $w$ to output $y$ is attenuated. The degree of the effect of disturbance attenuation is characterized by the value of $\gamma$. The smaller the value of $\gamma$, the stronger the corresponding effect. If $\gamma$ reaches its minimum, then we can get the so-called optimal robust control. However, if the value of $\gamma$ is too small and less than an existing minimum, the NDDAP has no solution.
Generally speaking, it is difficult to solve the NDDAP of system (1) thoroughly. Up to now, only for a few of simple cases, such as lower-triangular structure model [16], the problem can be solved. In this paper, we are going to develop a new recursive method to solve the NDDAP of a large scale nonlinear system and apply it to power systems.

\section{Multi-Machine System Model With Disturbances}

An $n$-generator power system with disturbances can be described in the form

$$
\begin{aligned}
& \left\{\begin{aligned}
\dot{\delta}_{i}(t)= & \omega_{i}(t)-\omega_{0} \\
\dot{\omega}_{i}(t)= & \frac{\omega_{0}}{M_{i}} P_{m i}-\frac{D_{i}}{M_{i}}\left(\omega_{i}(t)-\omega_{0}\right)-\frac{\omega_{0}}{M_{i}} P_{e i}+\frac{\omega_{0}}{M_{i}} \varepsilon_{1 i} \\
\dot{E}_{q i}^{\prime}(t)= & -\frac{1}{T_{d 0 i}}\left[E_{q i}^{\prime}(t)+I_{d i}\left(x_{d i}-x_{d i}^{\prime}\right)\right]+\frac{1}{T_{d 0 i}} V_{f i}(t) \\
& +\frac{1}{T_{d 0 i}} \varepsilon_{2 i}
\end{aligned}\right. \\
& z_{i}=\left[\begin{array}{l}
q_{1 i}\left(\delta_{i}(t)-\delta_{0 i}\right) \\
q_{2 i}\left(\omega_{i}(t)-\omega_{0}\right)
\end{array}\right] \\
& 1 \leq i \leq n
\end{aligned}
$$

where

$$
P_{e i}=E_{q i}^{\prime} \sum_{j=1}^{n} E_{q j}^{\prime}\left(B_{i j} \sin \left(\delta_{i}-\delta_{j}\right)+G_{i j} \cos \left(\delta_{i}-\delta_{j}\right)\right)
$$

is active power;

$$
I_{d i}=\sum_{j=1, j \neq i}^{n} E_{q j}^{\prime}\left(G_{i j} \sin \left(\delta_{i}-\delta_{j}\right)-B_{i j} \cos \left(\delta_{i}-\delta_{j}\right)\right)
$$

is $d$-axis current; $z_{i}$ is regulation output; $\varepsilon_{1 i}$ denotes torque disturbance acting on rotating shaft of the generator set; $\varepsilon_{2 i}$ denotes the electromagnetism disturbance entering the excitation winding described by the third equation of (3); $q_{1 \mathrm{i}}$ and $q_{2 \mathrm{i}}$ are weighting constants to be determined. Since the error of rotor speed is smaller than that of rotor angle, weighting factor $q_{2 \mathrm{i}}$ is selected larger than $q_{1 \mathrm{i}}$. In the meantime, $q_{1 \mathrm{i}}$ should not be too small because it reflects directly the power angle transients. For our 6-machine test system (see Section IV) we use $0.3 \leq q_{1 \mathrm{i}} \leq 0.4$ and $0.6 \leq q_{2 \mathrm{i}} \leq 0.7$. In the selected interval the system behavior is almost the same. If necessary, genetic algorithm can be used to optimize $q_{1 \mathrm{i}}$ and $q_{2 \mathrm{i}}$. Other notations appeared in (3) are the same as those in [10].

For system (3), the problem defined in Section II is going to be solved. A nonlinear decentralized disturbance attenuation excitation control signal $V_{f i}(i=1,2, \ldots, n)$ will be developed, so that the transient stability as well as dynamic performances of the corresponding closed-loop power system is improved effectively in spite of the existence of the disturbances $\varepsilon_{1 \mathrm{i}}$ and $\varepsilon_{2 \mathrm{i}}$ in (3).

To begin with, set pre-feedback

$$
V_{f i}=I_{d i}\left(x_{d i}-x_{d i}^{\prime}\right)+E_{q i}^{\prime}+v_{i} T_{d o i}
$$

where the introduced variable $v_{i}$ could be regarded as a new input of the system. 
Substituting (4) into (3) yields

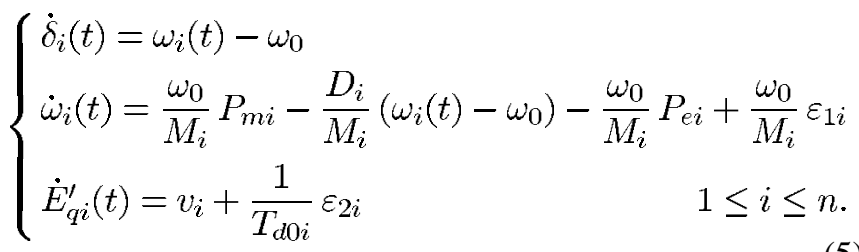

Denote

$$
\begin{gathered}
x_{i}=\left[\begin{array}{l}
x_{1 i} \\
x_{2 i} \\
x_{3 i}
\end{array}\right]=\left[\begin{array}{l}
\delta_{i}-\delta_{0 i} \\
\omega_{i}-\omega_{0} \\
E_{q i}^{\prime}
\end{array}\right], \quad a_{1 i}=\frac{\omega_{0}}{M_{i}}, \quad a_{2 i}=-\frac{D_{i}}{M_{i}} \\
a_{3 i}=\frac{1}{T_{d o i}}, \quad P_{m i}=P_{m o i}, \quad \varepsilon_{i}=\left[\begin{array}{l}
\varepsilon_{1 i} \\
\varepsilon_{2 i}
\end{array}\right]
\end{gathered}
$$

Choose the regulation output as

$$
z_{i}=\left[\begin{array}{l}
q_{1 i} x_{1 i} \\
q_{2 i} x_{2 i}
\end{array}\right] \quad q_{1 i} \geq 0, q_{2 i} \geq 0, q_{1 i}+q_{2 i}=1
$$

System (5) can be written as:

$$
\begin{cases}\dot{x}_{1 i}=x_{2 i} & \\
\dot{x}_{2 i}=a_{1 i}\left(P_{m o i}-P_{e i}\right)+a_{2 i} x_{2 i}+a_{1 i} \varepsilon_{1 i} \\
\dot{x}_{3 i}=v_{i}+a_{3 i} \varepsilon_{2 i} & 1 \leq i \leq n \\
z_{i}=\left[\begin{array}{l}
q_{1 i} x_{1 i} \\
q_{2 i} x_{2 i}
\end{array}\right] & \end{cases}
$$

For (8), in order to construct desired feedback control law, we consider the new input variable $v_{i}$ in (4) as a function of $x_{i}$

$$
v_{i}=\beta_{i}\left(x_{1 i}, x_{2 i}, x_{3 i}\right)
$$

and define the following coordinate transformation

$$
\left\{\begin{array}{l}
\hat{x}_{1 i}=x_{1 i} \\
\hat{x}_{2 i}=k_{i} x_{1 i}+x_{2 i} \\
\hat{x}_{3 i}=\phi_{i}\left(x_{1 i}, x_{2 i}, x_{3 i}\right) 1 \leq i \leq n
\end{array}\right.
$$

where $k_{i}>0$ is a given number and $\phi_{i}(\cdot)$ is a smooth function to be designed.

Our goal is to find $v_{i}=\beta_{i}\left(x_{1 i}, x_{2 i}, x_{3 i}\right)$ and $\phi_{i}\left(x_{1 i}\right.$, $x_{2 i}, x_{3 i}$ ) such that the inequality

$$
\int_{0}^{T}\|z\|^{2} d t \leq \gamma^{2} \int_{0}^{T}\|\varepsilon\|^{2} d t+V\left(x_{0}\right) T>0 V\left(x_{0}\right) \geq 0
$$

holds. Here $V(\cdot)$ is the storage function to be constructed, $x_{0}$ is the initial state of (8), $\gamma$ is a prescribed positive number, $z=$ $\left[z_{1}^{T} \cdots z_{n}^{T}\right]$ and $\varepsilon=\left[\varepsilon_{1}^{T} \cdots \varepsilon_{n}^{T}\right]$.

Inequality (11) is the dissipative inequality of the system, which shows that system (8) has an $L_{2}$-gain from disturbance $\varepsilon$ to output $z$ which is less than or equal to $\gamma$.

Now let us seek for a storage function $V(x): R^{n} \rightarrow R$, which satisfies dissipative inequality (11). As a consequence, a desired function $\phi_{i}(\cdot)$ in (10) and the function (9) will be achieved in the recursive design procedure, and the storage function $V(x)$, in fact, will play the role of a Lyapunov function of the corresponding closed-loop system. Our algorithm will involve three main steps.

Step 1: Let

$$
V_{1 i}\left(\hat{x}_{1 i}, \hat{x}_{2 i}\right)=\frac{\sigma_{i}}{2} \hat{x}_{1 i}^{2}+\frac{1}{2} \hat{x}_{2 i}^{2}
$$

where $\sigma_{i}$ is a positive number to be selected, and $\hat{x}_{1 i}$ and $\hat{x}_{2 i}$ have been defined by (10). From (10) we have

$$
\left\{\begin{array}{l}
\dot{\hat{x}}_{1 i}=-k_{i} x_{1 i}+\hat{x}_{2 i} \\
\dot{\hat{x}}_{2 i}=k_{i} \dot{x}_{1 i}+\dot{x}_{2 i}=\left(k_{i}+a_{2 i}\right) x_{2 i}+a_{1 i} \bar{P}_{e i}+a_{1 i} \varepsilon_{1 i} \\
\dot{\hat{x}}_{3 i}=\frac{\partial \phi_{i}}{\partial x_{1 i}} \dot{x}_{1 i}+\frac{\partial \phi_{i}}{\partial x_{2 i}} \dot{x}_{2 i}+\frac{\partial \phi_{i}}{\partial x_{3 i}} \dot{x}_{3 i}
\end{array}\right.
$$

where $\bar{P}_{e i}=P_{m o i}-P_{e i}, 1 \leq i \leq n$.

Then we introduce a function denoted by $H_{1 i}$ which is defined as

$$
H_{1 i}=\left.\dot{V}_{1 i}\right|_{(13)}+\frac{1}{2}\left\{\left\|z_{i}\right\|^{2}-\gamma_{i}^{2}\left\|\varepsilon_{1 i}\right\|^{2}\right\}
$$

where $\left.\dot{V}_{1 i}\right|_{(13)}$ denotes the differential of function $V_{1 i}\left(\hat{x}_{1 i}, \hat{x}_{2 i}\right)$ given by (12) with respect to time $t$ along the equation (13). From (13) we know that (14) can be written as

$$
\begin{aligned}
H_{1 i}= & \sigma_{i} \hat{x}_{1 i}\left(-k_{i} \hat{x}_{1 i}+\hat{x}_{2 i}\right)+\hat{x}_{2 i}\left(\left(k_{i}+a_{2 i}\right) x_{2 i}+a_{1 i} \bar{P}_{e i}\right) \\
& +\hat{x}_{2 i} a_{1 i} \varepsilon_{1 i}+\frac{1}{2}\left\|z_{i}\right\|^{2}-\frac{\gamma_{i}^{2}}{2}\left\|\varepsilon_{1 i}\right\|^{2}
\end{aligned}
$$

where $\gamma_{i}>0$ is a prescribed number and $1 \leq i \leq n$.

According to (7) and the coordinate transformation defined in $(10)$, we have

$$
\frac{1}{2}\left\|z_{i}\right\|^{2}=\frac{1}{2}\left\{\left(q_{1 i}^{2}+q_{2 i}^{2} k_{i}^{2}\right) \hat{x}_{1 i}^{2}+q_{2 i}^{2} \hat{x}_{2 i}^{2}-2 q_{2 i}^{2} k_{i} \hat{x}_{1 i} \hat{x}_{2 i}\right\} .
$$

By substituting (16) into (15) and making some algebraic manipulation, we have

$$
\begin{aligned}
H_{1 i}= & -e_{i} \hat{x}_{1 i}^{2}-\frac{1}{4}\left(\gamma_{i} \varepsilon_{1 i}-\frac{2}{\gamma_{i}} a_{1 i} \hat{x}_{2 i}\right)^{2}-\frac{\gamma_{i}^{2}}{4} \varepsilon_{1 i}^{2} \\
& +\hat{x}_{2 i}\left(\mu_{1 i} x_{1 i}+\mu_{2 i} x_{2 i}+a_{1 i} \bar{P}_{e i}\right)
\end{aligned}
$$

where

$$
\begin{aligned}
e_{i} & =\sigma_{\mathrm{i}} k_{i}-\frac{1}{2} q_{1 i}^{2}-\frac{1}{2} q_{2 i}^{2} k_{i}^{2} \\
\mu_{1 i} & =\frac{1}{\gamma_{i}^{2}} k_{i} a_{1 i}^{2}+\sigma_{i}-\frac{1}{2} k_{i} q_{2 i}^{2} \\
\mu_{2 i} & =\frac{a_{1 i}^{2}}{\gamma_{i}^{2}}+a_{2 i}+k_{i}+\frac{1}{2} q_{2 i}^{2} .
\end{aligned}
$$

Now, we define the last component of the new coordinates $\hat{x}_{3 i}$ in (10) as a linear function of $x_{1 i}, x_{2 i}$ and $x_{3 i}$ taking the form

$$
\hat{x}_{3 i}=\phi_{i}\left(x_{1 i}, x_{2 i}, x_{3 i}\right)=\mu_{1 i} x_{1 i}+\mu_{2 i} x_{2 i}-x_{3 i} .
$$

Since the second term of the right side of (17) is negative, and considering (21), we can see that the inequality

$$
H_{1 i} \leq-e_{1 i} x_{1 i}^{2}+\hat{x}_{2 i} \hat{x}_{3 i}-\frac{\gamma_{i}^{2}}{4} \varepsilon_{1 i}^{2}+\hat{x}_{2 i}\left(a_{1 i} \bar{P}_{e i}+x_{3 i}\right)
$$


Step 2: Based on Step 1, construct the nonnegative function

$$
V_{2 i}\left(\hat{x}_{1 i}, \hat{x}_{2 i}, \hat{x}_{3 i}\right)=\frac{\sigma_{i}}{2} \hat{x}_{1 i}^{2}+\frac{1}{2} \hat{x}_{2 i}^{2}+\frac{1}{2} \hat{x}_{3 i}^{2}
$$

and define

$$
\begin{aligned}
H_{2 i}= & \left.\dot{V}_{2 i}\right|_{(13)}+\frac{1}{2}\left(\left\|z_{i}\right\|^{2}-\gamma_{i}^{2}\left\|\varepsilon_{i}\right\|^{2}\right) \\
= & \left(\left.\dot{V}_{1 i}\right|_{(13)}+\frac{1}{2}\left(\left\|z_{i}\right\|^{2}-\gamma_{i}^{2}\left\|\varepsilon_{1 i}\right\|^{2}\right)\right) \\
& -\frac{1}{2} \gamma_{i}^{2} \varepsilon_{2 i}^{2}+\hat{x}_{3 i} \dot{\hat{x}}_{3 i}
\end{aligned}
$$

where $\left.\dot{V}_{2 i}\right|_{(13)}$ denotes the differential of function $V_{2 i}\left(\hat{x}_{1 i}, \hat{x}_{2 i}, \hat{x}_{3 i}\right)$ along the state equation (13).

Since

$$
\dot{\hat{x}}_{3 i}=\dot{\phi}_{i}\left(x_{1 i}, x_{2 i}, x_{3 i}\right)=\frac{\partial \phi_{i}}{\partial x_{1 i}} \dot{x}_{1 i}+\frac{\partial \phi_{i}}{\partial x_{2 i}} \dot{x}_{2 i}+\frac{\partial \phi_{i}}{\partial x_{3 i}} \dot{x}_{3 i}
$$

(24) can be rewritten as

$$
\begin{aligned}
H_{2 i}= & \left.\dot{V}_{1 i}\right|_{(13)}+\frac{1}{2}\left(\left\|z_{i}\right\|^{2}-\gamma_{i}^{2}\left\|\varepsilon_{1 i}\right\|^{2}\right)-\frac{1}{2} \gamma_{i}^{2} \varepsilon_{2 i}^{2} \\
& +\hat{x}_{3 i}\left(\frac{\partial \phi_{i}}{\partial x_{1 i}} \dot{x}_{1 i}+\frac{\partial \phi_{i}}{\partial x_{2 i}} \dot{x}_{2 i}+\frac{\partial \phi_{i}}{\partial x_{3 i}} \dot{x}_{3 i}\right) .
\end{aligned}
$$

Similar to Step 1, there is no difficulty to prove

$$
\begin{aligned}
H_{2 i} \leq & -e_{1 i} \hat{x}_{1 i}^{2}-\frac{1}{4}\left(\gamma_{i} \varepsilon_{1 i}-\frac{2 \hat{x}_{3 i} \mu_{2 i} a_{1 i}}{\gamma_{i}}\right)^{2} \\
& -\frac{1}{2}\left(\gamma_{i} \varepsilon_{2 i}+\frac{\hat{x}_{3 i} a_{3 i}}{\gamma_{i}}\right)^{2}+\hat{x}_{2 i}\left(a_{1 i} \bar{P}_{e i}+x_{3 i}\right) \\
+ & \hat{x}_{3 i}\left\{k_{i} x_{1 i}+\left(1+\mu_{1 i}+\mu_{2 i} a_{2 i}\right) x_{2 i}\right. \\
& \left.+\frac{\hat{x}_{3 i}}{\gamma_{i}^{2}}\left(\mu_{2 i}^{2} a_{1 i}^{2}+\frac{a_{3 i}^{2}}{2}\right)+\mu_{2 i} a_{1 i} \bar{P}_{e i}-v_{i}\right\}
\end{aligned}
$$

Since the second and third terms of the right hand side of (26) are both negative, the following inequality must hold

$$
\begin{aligned}
& H_{2 i} \leq-e_{1 i} \hat{x}_{1 i}^{2}+\hat{x}_{2 i}\left(a_{1 i} \bar{P}_{e i}+x_{3 i}\right) \\
&+\hat{x}_{3 i}\left\{k_{i} x_{1 i}+\left(1+\mu_{1 i}+\mu_{2 i} a_{2 i}\right) x_{2 i}+\frac{\hat{x}_{3 i}}{\gamma_{i}^{2}}\right. \\
&\left.\cdot\left(\mu_{2 i}^{2} a_{1 i}^{2}+\frac{a_{3 i}^{2}}{2}\right)+\mu_{2 i} a_{1 i} \bar{P}_{e i}-v_{i}\right\}
\end{aligned}
$$

Therefore in (27) we set

$$
\begin{aligned}
v_{i}= & k_{i} x_{1 i}+\left(1+\mu_{1 i}+\mu_{2 i} a_{2 i}\right) x_{2 i}+\mu_{2 i} a_{1 i} \bar{P}_{e i} \\
& \left.+\frac{\hat{x}_{3 i}}{\gamma_{i}^{2}}\left(\mu_{2 i}^{2} a_{1 i}^{2}+\frac{a_{3 i}^{2}}{2}\right)+\frac{\hat{x}_{2 i}}{\hat{x}_{3 i}}\left(a_{1 i} \bar{P}_{e i}+x_{3 i}\right)\right\}
\end{aligned}
$$

it must have

$$
H_{2 i} \leq-e_{1 i} \hat{x}_{1 i}^{2}
$$

In view of (18), if let

$$
\sigma_{i} \geq \frac{1}{2 k_{i}}\left(q_{1 i}^{2}+q_{2 i}^{2} k_{i}^{2}\right)
$$

then

$$
e_{1 i} \geq 0
$$

From (25) and (29), we have

$$
\left.\dot{V}_{2 i}\right|_{(13)}+\frac{1}{2}\left\{\left\|z_{i}\right\|^{2}-\gamma^{2}\left\|\varepsilon_{i}\right\|^{2}\right\} \leq-e_{1 i} \hat{x}_{1 i}^{2} \leq 0 .
$$

Step 3: For the whole multi-machine system, we define the storage function as

$$
V\left(x_{1}, x_{2}, \ldots, x_{n}\right)=\sum_{i=1}^{n} V_{2 i}
$$

then

$$
\begin{aligned}
\dot{V} & =\left.\sum_{i=1}^{n} \dot{V}_{2 i}\right|_{(13)} \leq \frac{1}{2} \sum_{i=1}^{n}\left\{\gamma^{2}\left\|\varepsilon_{i}\right\|^{2}-\left\|z_{i}\right\|^{2}\right\} \\
& =\frac{1}{2}\left(\gamma^{2}\|\varepsilon\|^{2}-\|z\|^{2}\right)
\end{aligned}
$$

where $\gamma=\max _{1 \leq i \leq n}\left\{\gamma_{i}\right\}$.

Inequality (32) implies

$2 V(x(T))-2 V(x(0)) \leq \int_{0}^{T}\left(\gamma^{2}\|\varepsilon\|^{2}-\|z\|^{2}\right) d t \quad \forall T>0$.

Inequality (33) is called dissipative inequality of system (3).

Because $V(x(T))$ is nonnegative, (33) says

$$
\int_{0}^{T}\|z\|^{2} d t \leq \gamma^{2} \int_{0}^{T}\|\varepsilon\|^{2} d t+2 V(x(0)) .
$$

Comparing (34) with (2) and remembering the pre-feedback (4), we can see that the $L_{2}$-gain of system (3) from disturbance $\varepsilon$ to output $z$ is less than or equal to a prescribed number $\gamma$. This means that the problem of nonlinear disturbance attenuation of a power system, as stated in Section II, has been solved by exploiting the feedback strategy (4). Now, we can summarize the acquired final result in a formal statement.

Statement of Results: Consider multi-machine system (3), its nonlinear decentralized disturbance attenuation excitation control for each generator can be implemented by using the feedback strategy in the expressions as

$$
\left\{\begin{array}{l}
V_{f i}=I_{d i}\left(x_{d i}-x_{d i}^{\prime}\right)+E_{q i}^{\prime}+v_{i} T_{d o i} \\
v_{i}=k_{i} \Delta \delta_{i}+\left(1+\mu_{1 i}+\mu_{2 i} a_{2 i}\right) \\
\cdot \Delta \omega_{i}+\mu_{2 i} a_{1 i}\left(P_{m 0 i}-P_{e i}\right) \\
+\frac{\mu_{1 i} \Delta \delta_{i}+\mu_{2 i} \Delta \omega_{i}-E_{q i}^{\prime}}{\gamma_{i}^{2}}\left(\mu_{2 i}^{2} a_{1 i}^{2}+\frac{a_{3 i}^{2}}{2}\right) \\
+\frac{k_{i} \Delta \delta_{1 i}+\Delta \omega_{i}}{\mu_{1 i} \Delta \delta_{i}+\mu_{2 i} \Delta \omega_{i}-E_{q i}^{\prime}}\left(a_{1 i}\left(P_{m 0 i}-P_{e i}\right)+E_{q i}^{\prime}\right)
\end{array}\right.
$$

where $\Delta \delta_{i}=\delta_{i}-\delta_{0 i}, \Delta \omega_{i}=\omega_{i}-\omega_{0}$ and $E_{q i}^{\prime}$ are the state variables of the $i$ th generator; $k_{i}, \mu_{1 i}, \mu_{2 i}, a_{1 i}, a_{2 i}$ and $a_{3 i}$ are given in (10), (19), (20), and (6).

Remarks:

1) In the derived control law (35) for generator $i$, all the variables and parameters are just related to the same generator, so it is clear that the proposed control law is indeed a decentralized one. 
2) From power system dynamics [2], we know that

$$
\begin{gathered}
E_{q i}^{\prime}+\left(x_{d i}-x_{d i}^{\prime}\right) I_{d i}=E_{q i}, \\
\Delta \delta_{i}=\int_{0}^{t} \Delta \omega_{i} d \tau, \quad P_{m 0 i}=P_{e 0 i} \\
E_{q i}=V_{t i}+\frac{x_{d i} Q_{e i}}{V_{t i}} \quad E_{q i}^{\prime} \approx V_{t i}+\frac{x_{d i}^{\prime} Q_{e i}}{V_{t i}}
\end{gathered}
$$

where $V_{t i}$ is the terminal voltage of generator $i ; Q_{e i}$ is generator reactive power output. As a result, the control strategy given by (35) can be rewritten as

$$
\begin{aligned}
& V_{f i}=V_{t i}+\frac{x_{d i} Q_{e i}}{V_{t i}}+T_{d o i} \\
& \left\{k_{i} \int_{0}^{t} \Delta \omega_{i} d \tau+\left(1+\mu_{1 i}+\mu_{2 i} a_{2 i}\right) \Delta \omega_{i}\right. \\
& +\mu_{2 i} a_{1 i}\left(P_{e 0 i}-P_{e i}\right) \\
& +\frac{\mu_{1 i} \int_{0}^{t} \Delta \omega_{i} d \tau+\mu_{2 i} \Delta \omega_{i}-V_{t i}-x_{d i}^{\prime} Q_{e i} / V_{t i}}{\gamma_{i}^{2}} \\
& \cdot\left(\mu_{2 i}^{2} a_{1 i}^{2}+\frac{a_{3 i}^{2}}{2}\right) \\
& +\frac{k_{i} \int_{0}^{t} \Delta \omega_{i} d \tau+\Delta \omega_{i}}{\mu_{1 i} \int_{0}^{t} \Delta \omega_{i} d \tau+\mu_{2 i} \Delta \omega_{i}-V_{t i}-x_{d i}^{\prime} Q_{e i} / V_{t i}} \\
& \left.\cdot\left(a_{1 i}\left(P_{e 0 i}-P_{e i}\right)+V_{t i}+x_{d i}^{\prime} Q_{e i} / V_{t i}\right)\right\}
\end{aligned}
$$

where all the variables are local measurements. Control law (36) looks somewhat complicated, however there is no difficulty in digital implementation.

3) The proposed control law can make the corresponding closed-loop system possesses a $L_{2}$-gain from disturbance to regulation output which is less than or equal to a prescribed number $\gamma$. That is to say the influence of disturbance on output of the system is weakened under the action of the proposed control law as we mentioned in Section II.

4) The back-stepping technology [19] is applied to the disturbance attenuation control design. The essence of the approach is to construct a storage function for the control system. As we mentioned above the new approach first pre-sets an imperfect "storage function" for the first two sub-equations of state equation (3), then extend it to the last sub-equation of (3) and yield a perfect storage function. So we call it a "recursive" method.

5) The work reported above is indeed a continuation of previous research work [5], [6], [10], [11], [17].

\section{Computer Test Results}

A 6-machine power system is used for computer test (Fig. 1). The system data is listed in Appendix A where machine 6 of

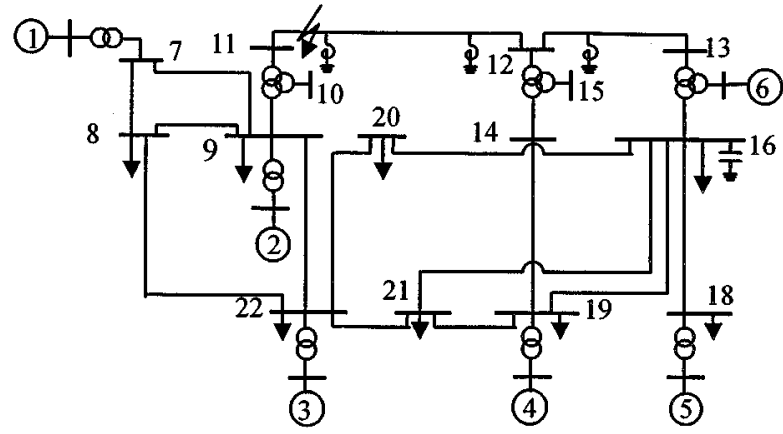

Fig. 1. Test system diagram.

the system is a synchronous condenser with a conventional PID-type excitation controller. In the time simulation, the generators' models are the same as in (3) and there are no turbine governors. In order to investigate the effectiveness of the suggested controller in improving transient stability, comparisons are made to several different types of excitation controllers.

In the test we set $k_{i}=2, \sigma_{i}=1, q_{1 i}=0.4, q_{2 i}=0.6$ and $\gamma_{i}=10$.

The selection of these parameters is based on the following considerations. For $k_{i}$ and $\sigma_{i}$, we select them based on the inequality $e_{i} \geq 0$. In order to avoid high gain, we usually select the smallest values of $k_{i}$ and $\sigma_{i}$ which satisfy $e_{i} \geq 0$. And the smallest $k_{i}$ and $\sigma_{i}$ are approximately equal to 2 and 1 . The selection of $q_{1 \mathrm{i}}$ and $q_{2 \mathrm{i}}$ has been explained in (3). The values of $q_{1 i}$ and $q_{2 i}$ are set to be 0.4 and 0.6 , respectively. Parameter $\gamma_{I}$ characterizes the degree of the effect of disturbance attenuation. The smaller the value of $\gamma_{i}$, the better the disturbance attenuation effect. Of course, as stated in Section II, the value of $\gamma_{i}$ should not be too small for secure NDDAP solution. For the 6-machine test system, the smallest $\gamma_{i}$ is approximately 10 .

Four cases are studied. They are:

Case 1: Generators 2 to 5 are installed conventional PSSs with their transfer functions given in Appendix B-1; Case 2: Generators 2 to 5 are equipped with linear optimal excitation control designed by LQR approach with feedback gains given in Appendix B-2; Case 3: The same generators are equipped with nonlinear optimal excitation controllers (NOEC) [5]; and Case 4: The generators are equipped with the nonlinear decentralized disturbance excitation controllers (NDDEC) proposed in this paper using the control law given in (36).

System transients are stimulated by a three-phase short circuit fault occurred on line 11-12 close to bus 11 (see Fig. 1) and cleared by tripping the faulted line in $0.1 \mathrm{~s}$. The pre-fault power flow in line 11-12 is 3.66-j0.62 pu. The simulation results for the 4 cases are shown in Figs. 2-5, respectively, where generator rotor angle response to the fault is plotted.

We can see from Figs. 2 and 3 that if linear PSS or LOEC is used, the system loses its synchronism soon after the fault occurs. However the system remains stable when NOEC or NDDEC is applied (see Figs. 4 and 5). Comparing Fig. 5 with Fig. 4, we can see that when NDDEC scheme is used, the corresponding rotor dynamics is better than that of NOEC scheme a little. Besides NDDEC has better robustness, which is significant to real power system applications. Figs. 6-9 show 


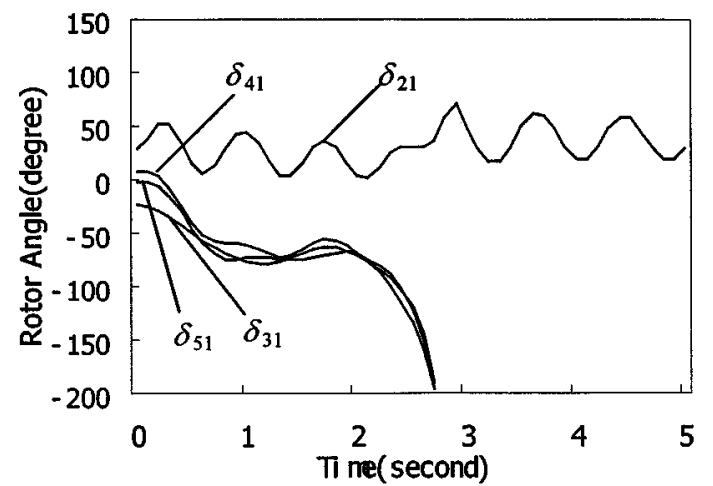

Fig. 2. Dynamic response of the system with PSS.

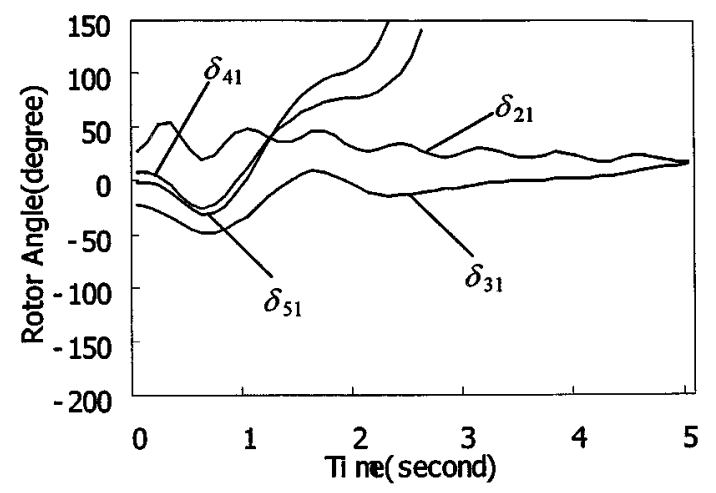

Fig. 3. Dynamic response of the system with LOEC.

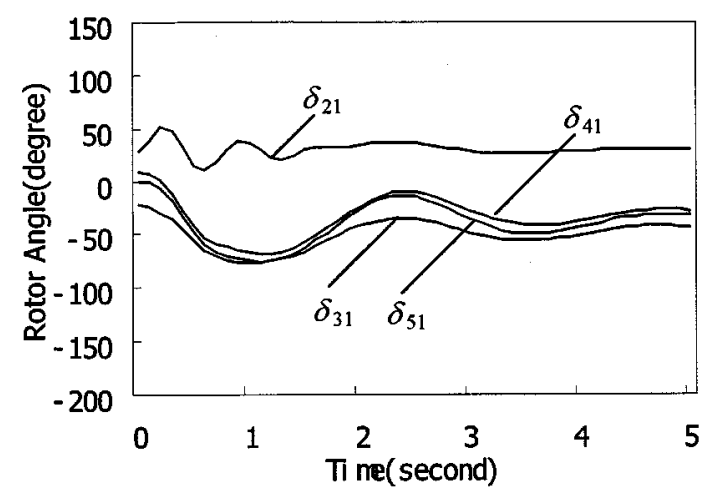

Fig. 4. Dynamic response of the system with NOEC.

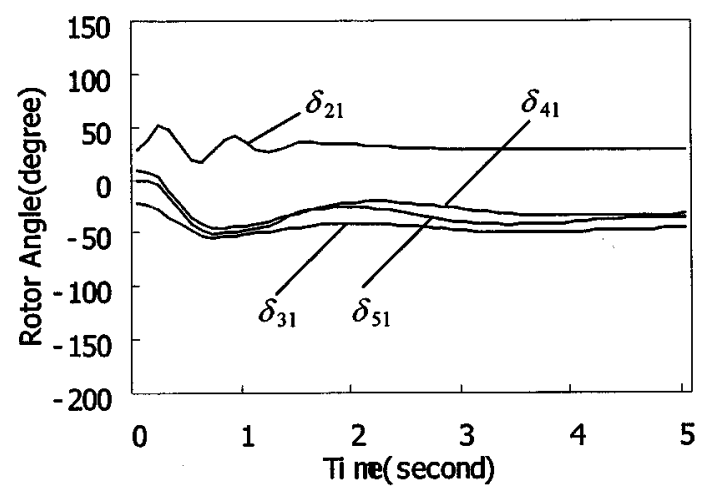

Fig. 5. Dynamic response of the system with NDDEC.

the excitation voltage $\mathrm{E}_{\mathrm{fd}}$ of the generator 2 under the four different controls for comparison.

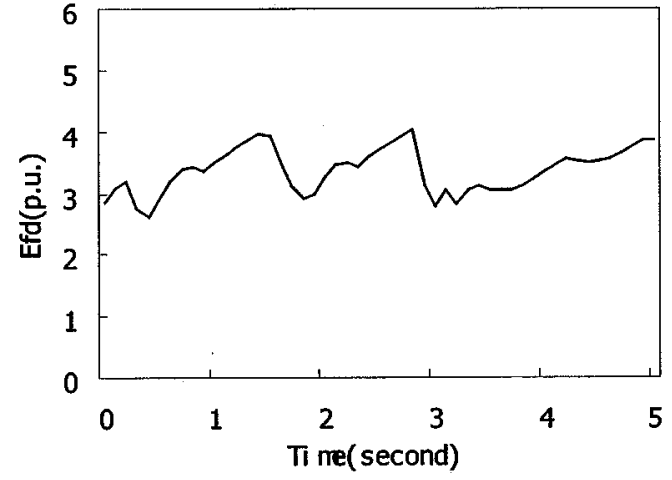

Fig. 6. Efd of the generator 2 with PSS.

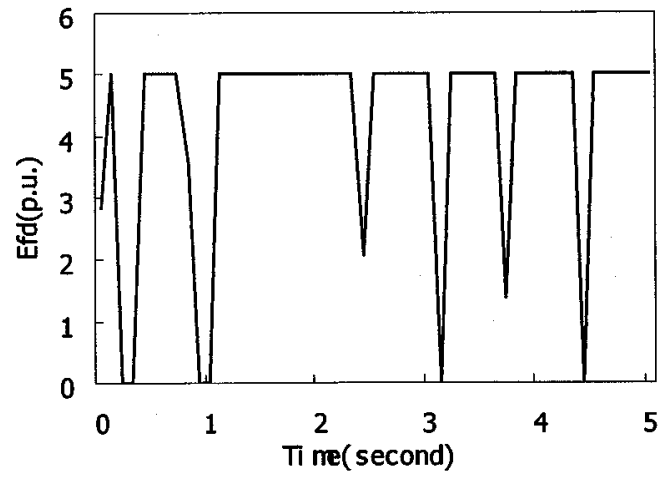

Fig. 7. Efd of the generator 2 with LOEC.

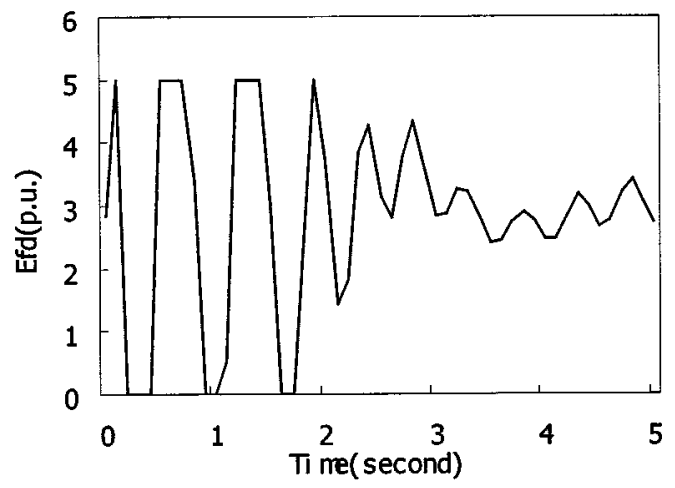

Fig. 8. Efd of the generator 2 with NOEC.

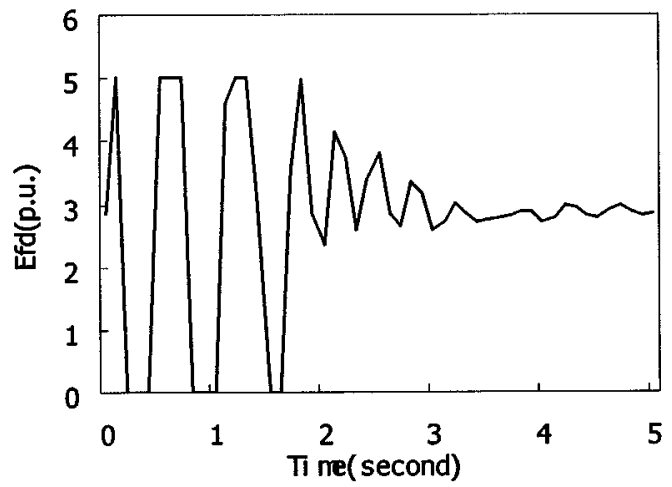

Fig. 9. Efd of the generator 2 with NDDEC.

Since the NOEC method is based on mathematical models with fixed structure and parameters without considering 
uncertainties, theoretically the NDDEC is superior to NOEC in robustness, just as the linear robust control is superior to linear optimal control (LOEC) in robustness, which has been verified by modern control theory strictly [17], [18]. We also observed this feature in other test cases which are not presented here for the limited length.

\section{CONCLUSION}

In this paper, a novel nonlinear decentralized disturbance attenuation (NDDAP) excitation control has been designed for multi-machine systems. Based on a recursive design approach, solution of the dissipative inequality (31) of multi-machine systems can be reached without performing system linearization. The obtained control law (34) has following apparent advantages.

The control law is of robustness in the sense of $L_{2}$-gain. The control strategy is decentralized and independent to networks parameters. It is easy to implement the control law using microcomputer, since all the variables appearing in the expression of this control law are local measurements. The control is nonlinear and much effective in enhancing system transient stability under large disturbances.

Computer test results from a 6-machine power system show clearly the effectiveness of the suggested nonlinear robust excitation control in improving transient stability and dynamic performances of power systems.

\section{APPENDIX A \\ SYSTEM DATA}

TABLE I

LINE AND TRANSFORMER PARAMETERS

\begin{tabular}{llccc}
\hline 14 & 19 & 0.0034 & 0.0200 & 0.0000 \\
16 & 16 & 0.0000 & -1.9930 & 0.0000 \\
17 & 16 & 0.0000 & 0.0010 & $1.027^{*}$ \\
16 & 18 & 0.0033 & 0.0333 & 0.0000 \\
16 & 19 & 0.0578 & 0.2180 & -0.1807 \\
16 & 20 & 0.0165 & 0.0662 & -0.2353 \\
16 & 21 & 0.0374 & 0.1780 & -0.1640 \\
19 & 21 & 0.0114 & 0.0370 & 0.0000 \\
20 & 22 & 0.0214 & 0.0859 & -0.3008 \\
21 & 22 & 0.0150 & 0.0607 & -0.2198 \\
\hline
\end{tabular}

TABLE II

POWER FLOW DATA

\begin{tabular}{ccccc}
\hline Bus & $\mathrm{P}_{\mathrm{g}}$ & $\mathrm{V}_{1} / \mathrm{Q}_{\mathrm{g}}{ }^{*}$ & $\mathrm{P}_{\text {load }}$ & $\mathrm{Q}_{\text {load }}$ \\
\hline 1 & 6.0 & 1.0 & 0.0 & 0.0 \\
2 & 6.0 & $3.2^{*}$ & 0.0 & 0.0 \\
3 & 3.1 & 1.0 & 0.0 & 0.0 \\
4 & 1.6 & $0.7^{*}$ & 0.0 & 0.0 \\
5 & 4.3 & $3.34^{*}$ & 0.0 & 0.0 \\
6 & -0.01 & 1.0 & 0.0 & 0.0 \\
8 & 0.0 & $0.0^{*}$ & 2.87 & 1.44 \\
9 & 0.0 & $0.0^{*}$ & 3.76 & 2.21 \\
16 & 0.0 & $0.0^{*}$ & 5.0 & 2.9 \\
18 & 0.0 & $0.0^{*}$ & 4.3 & 2.6 \\
19 & 0.0 & $0.0^{*}$ & 0.864 & 0.662 \\
20 & 0.0 & $0.0^{*}$ & 0.72 & 0.47 \\
21 & 0.0 & $0.0^{*}$ & 0.7 & 0.5 \\
22 & 0.0 & $0.0^{*}$ & 2.26 & 1.59 \\
\hline
\end{tabular}

TABLE III

SYNCHRONOUS MACHINE DATA

\begin{tabular}{cccccc}
\hline$x_{d}$ & $x_{q}$ & $x_{d}^{\prime}$ & $H$ & $D$ & $T_{d 0^{\prime}}$ \\
\hline 0.015 & 0.105 & 0.105 & 140.82 & 3.0 & 9.0 \\
0.321 & 0.321 & 0.0382 & 30.0 & 3.0 & 8.375 \\
0.138 & 0.0396 & 0.0396 & 79.5 & 3.0 & 7.24 \\
0.770 & 0.770 & 0.121 & 15.62 & 3.0 & 6.2 \\
0.306 & 0.306 & 0.048 & 39.2 & 3.0 & 6.2 \\
1.663 & 1.663 & 0.197 & 2.62 & 3.0 & 6.92 \\
\hline
\end{tabular}

All in per unit except that $H$ and $T_{d 0}{ }^{\prime}$ are in second.

\section{APPENDIX B \\ STABILIZER DATA}

1) PSS transfer function (input signal is $\Delta \omega$ )

Generator 2:

$G_{2}(s)=\frac{15.48}{(1+0.01 s)} \frac{3 s}{(1+3 s)}\left(\frac{1+0.43 s}{1+0.045 s}\right)^{2}$

Generator 3:

$G_{3}(s)=\frac{63.35}{(1+0.01 s)} \frac{3 s}{(1+3 s)}\left(\frac{1+0.42 s}{1+0.155 s}\right)^{2}$

Generator 4:

$G_{4}(s)=\frac{14.3}{(1+0.01 s)} \frac{3 s}{(1+3 s)}\left(\frac{1+0.34 s}{1+0.126 s}\right)^{2}$

Generator 5:

$G_{5}(s)=\frac{25.6}{(1+0.01 s)} \frac{3 s}{(1+3 s)}\left(\frac{1+0.436 s}{1+0.21 s}\right)^{2}$

The output limitation of each PSS is $\pm 5 \%$.

2) Linear optimal excitation control laws

Generator 2:

$V_{\mathrm{t} 2}=-73.8 \Delta V_{\mathrm{t} 2}-35.6 \Delta P_{\mathrm{e} 2}+5.8 \Delta \omega_{2}$

Generator 3:

$V_{\mathrm{t} 3}=-69.2 \Delta V_{\mathrm{t} 3}-23.3 \Delta P_{\mathrm{e} 3}+6.1 \Delta \omega_{3}$

Generator 4:

$V_{\mathrm{t} 4}=-62.9 \Delta V_{\mathrm{t} 4}-10.0 \Delta P_{\mathrm{e} 4}+2.3 \Delta \omega_{4}$

Generator 5:

$$
V_{\mathrm{t} \tilde{5}}=-58.4 \Delta V_{\mathrm{t} \tilde{5}}-8.0 \Delta P_{\mathrm{e} \tilde{5}}+1.6 \Delta \omega_{5} .
$$

\section{REFERENCES}

[1] V. Schaft, $L_{2}$-Gain and Passivity Techniques in Nonlinear Control. London: Springer, 1996.

[2] J. H. Anderson, "The control of a synchronous machine using optimal control theory," Proc. IEEE, vol. 90, pp. 25-35, 1971.

[3] Y. N. Yu, Electric Power Systems Dynamics. New York: Academic Press, 1983.

[4] F. P. deMello and C. Concordia, "Concepts of synchronous machine stability as affected by excitation control," IEEE Trans. PAS, pp. 316-329, Apr. 1969.

[5] Q. Lu and Y. Sun, "Nonlinear stabilizing control of multi-machine systems," IEEE Trans. Power Systems, vol. 4, no. 1, pp. 236-241, 1989.

[6] Q. Lu, Y. Sun, and G. K. F. Lee, "Nonlinear optimal excitation control for multi-machine systems," in IFAC Symposium on Power System Modeling and Control Application, Brussels, Sept. 1988.

[7] L. Gao, L. Chen, Y. Fan, and H. Ma, "A nonlinear control design for power systems," Automatica, vol. 28, no. 7, pp. 975-979, 1992.

[8] Y. Wang, D. J. Hin, L. Gao, and R. H. Middleton, "Transient stability enhancement and voltage regulation of power systems," IEEE Trans. Power Systems, vol. PWRS-8, pp. 620-627, 1993.

[9] C. A. King, J. W. Chapman, and M. D. Ilic, "Feedback linearizing excitation controller on a full-scale power system model," IEEE Trans. Power Systems, vol. PWRS-9, pp. 1102-1109, 1994. 
[10] Q. Lu, Y. Sun, Z. Xu, and T. Mochizuki, "Decentralized nonlinear optimal excitation control," IEEE Trans. Power Systems, vol. 11, no. 4, pp. 1957-1962, 1996.

[11] C. Sun, Z. Zhao, Y. Sun, and Q. Lu, "Design of nonlinear robust excitation control for multi-machine power systems," IEE Proc. on Gener. Trans. and Distrib., vol. 143, no. 3, pp. 253-257, 1996.

[12] Y. Wang, G. Guo, and D. J. Hin, "Robust decentralized nonlinear controller design for multi-machine power systems," Automatica, vol. 33, no. 9, pp. 1725-1733, 1997.

[13] H. Jiang, H. Cai, J. F. Dorsey, and Z. Qu, "Toward a globally robust decentralized control for large-scale power systems," IEEE Trans. Control Systems Technology, vol. 5, no. 3, pp. 309-319, 1997.

[14] J. W. Chapman, M. D. Ilic, A. C. King, L. Eng, and H. Kaufman, "Stabilizing a multi-machine power system via decentralized feedback linear excitation control," IEEE Trans. PWRS, vol. 8, no. 3, pp. 830-838, 1993.

[15] C. I. Byrnes, A. Isidori, and J. C. Willems, "Passity feedback equivalence and the global stabilization of minimum phase nonlinear systems," IEEE Trans. AC, vol. 36, no. 11, pp. 1228-1240, 1991 .

[16] D. B. Seto, A. M. Annaswamy, and J. Baillieul, "Adaptive control of nonlinear systems with a triangular structure," IEEE Trans. AC, vol. 39, no. 7, pp. 1411-1428, 1994.

[17] Q. Lu, Y. Sun, and S. Mei, Nonlinear System Control and Power System Dynamics. Boston/Dordrecht/London: Kluwer Academic Publishers, 2001.

[18] B. A. Francis, A Course in H Control Theory. New York: SpringerVerlag, 1987.

[19] M. Kristic, I. Kanellakopoulos, and P. Kokotovic, Nonlinear and Adaptive Control Design, NJ: Prentice-Hall, 1995.

Qiang Lu (SM' 85 ) graduated from the Graduate School of Tsinghua University, China, in 1963 and joined the faculty of the same university. He was a visiting scholar and a visiting professor in Washington University, St. Louis and Colorado State University, Ft. Collins, respectively, in 1984-1986 and a visiting professor of Kyushu Institute of Technology (KIT), Japan in 1993-1995. $\mathrm{He}$ is now a professor in Tsinghua University, and an academician of Chinese Academy of Science (1991-). His research interest is in nonlinear control theory applications in power system.
Shengwei Mei received the B.S. degree in mathematics from Xinjiang University, the M.S. degree in operations research from Tsinghua University, and the Ph.D. degree in automatic control from Chinese Academy of Sciences, Beijing, in 1984, 1989, and 1996, respectively. He is now an associate professor of Tsinghua University. His research interest is in control theory applications in power systems.

Wei $\mathbf{H u}$ is a Ph.D. candidate in Tsinghua University. His research interest is in nonlinear control theory applications in power systems.

Felix F. Wu received the Ph.D. degree from University of California at Berkeley (UCB). He is now a chair professor of electrical engineering and vice president of the University of Hong Kong. Prior to that he was a professor and vice-chair of Department of EECS, UCB. His research interests are electric energy industry restructuring, power system investment planning, design of modern control centers, distribution automation, distributed processing etc.

Yixin Ni received the B.Eng., M.Eng., and Dr.Eng. degrees all in electrical engineering, Tsinghua University, China. She was former professor and director of National Power System Lab, Tsinghua University and now with the University of Hong Kong. Her research interests are power system stability and control, FACTS, AI tech. applications in power systems and power market.

Tielong Shen received the B.S. and M.S. degrees in automatic control from Northeast Heavy Machinery Institute, China in 1982 and 1986, respectively, and the Ph.D. degree in mechanical engineering from Sophia University, Tokyo, Japan, 1992. Since April 1992, he has been a faculty member as an assistant Professor of the Department of Mechanical Engineering at Sophia University. 